ORIGINAL ARTICLE

\title{
Eczema and early solid feeding in preterm infants
}

\section{J Morgan, P Williams, F Norris, C M Williams, M Larkin, S Hampton}

See end of article for authors' affiliations

.....................

Correspondence to: Dr J Morgan, School of Biomedical and Life Sciences, University of Surrey, Guildford, Surrey GU2 $7 X \mathrm{H}, \mathrm{UK}$;

j.morgan@surrey.ac.uk

Accepted 3 June 2003

\begin{abstract}
Aims: To establish whether development of eczema is influenced by feeding practices in preterm infants, while taking account of confounding factors.

Methods: Data were assembled from 257 infants born prematurely and studied to 12 months post-term. Logistic regression analysis was performed to establish the association between feeding practices and eczema, allowing for potential confounding factors including the infants' gender, parental atopic status, social background, and parental smoking habits.

Results: For the development of eczema (with or without other symptoms) by 12 months post-term, the introduction of four or more solid foods by or before 17 weeks post-term was a significant risk (odds ratio 3.49). Male infants were at significantly higher risk (odds ratio 1.84). In addition, having non-atopic parents who introduced solid foods before 10 weeks post-term or having at least one atopic parent represented a significant risk scenario (odds ratio 2.94).

Conclusions: Early introduction of a diverse range of solid foods may predispose the preterm infant to eczema development by 12 months post-term. Furthermore, non-atopic parents who practice early as opposed to late introduction of solid foods may be exposing preterm infants to a greater risk of eczema by 12 months post-term.
\end{abstract}

$\mathrm{T}$ he European Society for Paediatric Allerogology and Clinical Immunology (ESPACI) Committee on Hypoallergenic Formulas and the European Society for Paediatric Gastroenterology, Hepatology and Nutrition (ESPGHAN) Committee on Nutrition has commented on current unresolved issues in the treatment and prevention of food allergy in early life. ${ }^{1}$ Reference to the role of solid feeding (weaning) practices in the cause or prevention of food allergy, including eczema, was limited.

Attempts to prevent or delay the development of allergic disorders in infants have been concentrated in three areas. First, exclusion diets during pregnancy may reduce the onset of allergy in high risk infants-that is, in infants where one or both parents are atopic, but the definitive study is still to be carried out. ${ }^{2}$ Second, maternal exclusion diets (in relation to milk and eggs) during lactation may benefit some breast fed high risk infants with eczema. ${ }^{3-5}$ There is also evidence that maternal exclusion diets during late gestation and lactation benefit the high risk infant. ${ }^{6-8}$ However, in a seven year follow up study of combined maternal and infant avoidance, ${ }^{9}$ perinatal maternal/infant food avoidance failed to modify atopic disease at 7 years of age compared with the infant feeding practices customarily followed. The third area has centred on the introduction of foods other than milk to the infants' diet; the weaning period. Kajaosaari and Saarinen ${ }^{10}$ and Wilson and colleagues $^{11}$ have shown in full term infants that the early introduction of solid food (before 3 months $^{10}$ or 15 weeks ${ }^{11}$ ) is associated with a higher prevalence of atopic dermatitis at 6 months and respiratory illness at 10 years, compared with the introduction of solid foods after 6 months $^{10}$ or 15 weeks. ${ }^{11}$ There is also evidence from New Zealand, in a 10 year follow up study of full term infants, that "children exposed to four or more different types of solid food before 4 months had risks of recurrent or chronic eczema that were 2.9 times those of children who were not exposed to early solid feeding". ${ }^{12}$

A review of the literature revealed that no data exist on evidence for appropriate solid feeding practices for preterm infants in the prevention of allergic disease development.
These infants, who could be both low birth weight and small for gestational age, may also be at greater risk than their full term counterparts for the development of atopic disorders because of their relatively immature immunological and physiological systems. ${ }^{13}$

We have reported previously on the development of allergies in a small cohort of small for gestational age infants ${ }^{14}$ and on the weaning practices of a cohort of preterm infants. ${ }^{15}$ Here we report the influence of solid food patterns on the development of eczema in the same cohort of preterm infants followed to 12 months corrected age (post-term), taking into account possible confounding factors including those previously associated with eczema development. The main aim of the study was to establish associations, if any, between feeding patterns in preterm infants and subsequent development of eczema, taking into account confounding factors.

\section{SUBJECTS AND METHODS Study population}

Subjects were recruited from a cross section of socioeconomic groups in the Surrey and Hampshire areas of southeast England using the Royal Surrey County Hospital (Guildford), St Peter's Hospital (Chertsey), and Frimley Park Hospital (Frimley). When a preterm birth ( $<37$ weeks gestational age) occurred in the delivery suite and provided that the baby was in reasonably good health, study midwives approached the mother after she returned to the maternity unit and informed her of the study, seeking her agreement and that of her partner to participate. Parents volunteered for the study after reading an information sheet; two to three weeks elapsed before written consent was sought and given. Any infant whose parents agreed to take part was recruited. Shortly after the mother/infant pair returned home they received a visit from a study midwife and a record was made of milk feeding practices during the time the infant was in hospital. Milk feeding practices in the hospital were in accordance with the wishes of the parents, though there was no requirement to give written consent for the feeding of cows' milk infant formula. 


\section{The questionnaires}

Once the mother/infant pair had been recruited onto the study, they were visited by the study midwives at home at regular intervals until the infant was 12 months post-term. Detailed demographic data were recorded for each infant and his/her family, including socioeconomic status, number of siblings, parents' tobacco use, the presence of furry pets, and central heating. Information on infant feeding practices was recorded via a detailed food frequency questionnaire, administered by the study midwives to the mother on enrolment and at four monthly intervals to 12 months post-term. The mothers were asked to recall the number and type of milk feeds given prior to the introduction of solid foods.

Infants were categorised into seven subgroups based on their milk feeding practices prior to solid food introduction: (1) exclusively breast fed, $\mathrm{n}=11$; (2) breast milk and hypoallergenic formula (Pepti-Junior, Nutricia, Trowbridge, Wilts BA14 0XQ), $\mathrm{n}=25$; (3) breast milk, hypoallergenic formula, and any cows' milk formula, $\mathrm{n}=22$; (4) breast fed at some point and receiving cows' milk formula less than one third of the time prior to the introduction of solid foods, $\mathrm{n}=31$; (5) breast fed at some point and receiving cows' milk formula one third to two thirds of the time prior to solid food introduction, $\mathrm{n}=68$; (6) breast fed at some point and receiving cows' milk formula more than two thirds of the time prior to solid food introduction, $\mathrm{n}=63$; and (7) exclusively fed cows' milk formula, $\mathrm{n}=37$.

Information was collected retrospectively on solid food practices and has been published elsewhere. ${ }^{15}$ The timings (corrected age) were recorded for the introduction of 12 individually listed foods: baby cereal, cheese, eggs, fish, fruit, meat, meat with vegetables, baby rice, baby rusks, vegetables, yoghurt, and other desserts. The timing of the earliest of these 12 foods was deemed to correspond to the age of solid food introduction. In view of the findings of Fergusson and colleagues, ${ }^{12}$ the timing of the introduction of four or more of these foods was extracted to investigate the possible chronological relation between diet and eczema.

\section{Outcome measures}

All infants were assessed at 12 months post-term by a paediatrician who was unaware of the atopic status of the infants' families. This assessment included a physical examination, an assessment of the presence (or absence) of recurrent skin, chest, nasal, eye, and gastrointestinal symptoms, and a structured parental interview by questionnaire, adapted from the Early Treatment of the Atopic Child (ETAC) Study. ${ }^{16}$ Eczema was defined as dryness, erythema, itching, or excoriation lasting for more than 30 days in one year, as diagnosed by the family general practitioner. Lower respiratory symptoms were defined as persistent recurrent nocturnal cough disturbing sleep on more than three consecutive nights or a wheeze of any duration. Upper respiratory symptoms were defined as nasal symptoms when recurrent sneezing, runny nose, blocked nose, or any two of these three symptoms lasting longer than two weeks in an episode were reported to the study paediatrician. Eye symptoms were defined as watery, itchiness, redness, or swollen eyelids for more than 48 hours in any one episode, not associated with infection. Gastrointestinal symptoms were defined as more than 48 hours of vomiting, diarrhoea (not associated with infection), or swelling of the lips of any duration.

\section{Confounding exposures}

Families were classified as either non-atopic or atopic. They were classified as atopic according to the following criteria: (1) by a closed question from a questionnaire, indicating a history of asthma/wheeze, eczema (skin rash), urticaria, reactions to foods (vomiting, diarrhoea, nausea), migraine, contact rashes, hay fever, and/or persistent runny nose; (2) skin prick tests (SPT) to common allergens (three trees, six grasses, cat, dog, house dust mite, two common moulds, cows' milk, and chicken egg) yielding a wheal greater than or equal to the histamine dihydrochloride $(10 \mathrm{mg} / \mathrm{ml})$ control response when the saline control gave a negative result; and (3) total serum IgE antibody level $>80 \mathrm{kU} / \mathrm{l}$. Families were classified as atopic if the mother or father (or both) was positive in at least two of the three criteria described.

Other potential confounding exposures included the infant's gender and gestational age, the number of siblings, smoking in the household, and the presence of furry pets.

\section{Ethical approval}

The study received approval from the North West Surrey Local Research Ethics Committee on l November 1996 (reference number PRO/107/96) and from the South West Local Research Ethics Committee on 12 November 1996 (reference number CT0793.107.166).

\section{Statistical analysis}

Simple descriptive statistics were derived from the overall group in the form of means, medians, standard deviations (SDs), and ranges. Comparisons between groups of infants from atopic and non-atopic backgrounds were undertaken using the $\chi^{2}$ or the Mann-Whitney U test. Differences were taken as significant if $\mathrm{p}<0.05$. Stepwise logistic regression was used to model eczema outcome against the confounding exposures described above, plus milk feeding and solid feeding regimens. In addition, possible interaction effects were evaluated between gender and time by which four or more foods had been introduced. Statistical analysis was undertaken using SAS version 6.

\section{RESULTS \\ Study population}

Three hundred and twenty nine infants were recruited onto the study from January 1997 to December 1998; 257 infants, $140(55 \%)$ male and 117 (45\%) female, belonging to 219 families completed the study-a withdrawal rate of $21.9 \%$. There were many stages at which infants were withdrawn from the study, ranging from immediately after the mother's written acceptance (at which point no demographic data were available regarding the parents) to a point in time at which it became evident that a parent had not undergone skin prick testing. Hence it was deemed impractical to carry out a comparison of the dropouts against the study group because of the varying levels of missing demographic data.

There were 183 singletons, 34 sets of twins, and two sets of triplets. The concordance between identical and non-identical siblings in the twins was not established. The majority of the parents $(54.8 \%)$ were classified into socioeconomic groups I and II as defined by the father's occupation.

The mean maternal age was 30.8 (SD 5.24) years and ranged from 17 to 47 years. The mean paternal age was 33.1 (SD 5.54) years and ranged from 18 to 51 years. The smoking habits of the parents varied during the study. Fourteen per cent of the mothers smoked when recruited onto the study, but by the end of the study $17.8 \%$ were smoking. Paternal smoking fell from $26.9 \%$ at the start of the study to $23.7 \%$ at the end. Fifty seven per cent of the households had pets (dogs, cats, and other furry animals). Forty seven per cent of infants had older siblings; their atopic status was unknown. Most of the homes of the infants $(96.5 \%)$ had gas, oil, or electric central heating. 
Table 1 Summary statistics of tests relating to atopic status for mothers and fathers from the 219 families

\begin{tabular}{|c|c|c|c|c|c|c|}
\hline \multirow[b]{2}{*}{ Test } & \multicolumn{2}{|c|}{ Positive } & \multicolumn{2}{|c|}{ Negative } & \multicolumn{2}{|c|}{ No assessment } \\
\hline & $n$ & $\%$ & $n$ & $\%$ & $n$ & $\%$ \\
\hline \multicolumn{7}{|l|}{ Mothers } \\
\hline SPT & 60 & 27.4 & 139 & 63.5 & 20 & 9.1 \\
\hline Questionnaire & 93 & 42.5 & 126 & 57.5 & 0 & 0 \\
\hline $\lg E \geqslant 80 \mathrm{kU} / \mathrm{l}$ & 52 & 23.7 & 136 & 62.1 & 31 & 14.2 \\
\hline Overall atopic status & 71 & 32.4 & 148 & 67.6 & 0 & 0 \\
\hline \multicolumn{7}{|l|}{ Fathers } \\
\hline SPT & 74 & 33.8 & 122 & 55.7 & 23 & 10.5 \\
\hline Questionnaire & 76 & 34.7 & 142 & 64.8 & 1 & 0.5 \\
\hline $\lg E \geqslant 80 \mathrm{kU} / \mathrm{l}$ & 59 & 26.9 & 112 & 51.1 & 48 & 21.9 \\
\hline Overall atopic status & 71 & 33.0 & 144 & 65.8 & 4 & 1.8 \\
\hline
\end{tabular}

\section{Atopic status of parents}

The atopic status of the mothers and fathers was based on results from the questionnaire, the SPT, and total serum IgE levels. Table 1 shows the results of these assessments. In the mothers, the results of the assessment from the questionnaire gave a noticeably higher prevalence of atopy than the SPT and IgE levels. The positive test results from the fathers were more compatible. Overall $32.4 \%$ of mothers and $33.0 \%$ of fathers were assessed as atopic.

As far as the results of the family data are concerned, there were four families $(1.8 \%)$ where classification could only be made for the mother; these four mothers were all atopic. There were $24(11.0 \%)$ families where both parents were classified as atopic, $43(19.6 \%)$ families where the mother was classified as atopic with the father non-atopic, and 47 $(21.0 \%)$ families where the father was classified as atopic with the mother non-atopic. In the remaining 101 (46.1\%) families both parents were classified as non-atopic. There was therefore at least one atopic parent in 118 (53.9\%) families.

\section{The infants}

The mean gestational age and mean birth weight of infants were similar in those having an atopic background compared with those from a non-atopic background (table 2). The infants with an atopic background comprised 75 (54.4\%) male and $63(45.7 \%)$ females; those with a non-atopic background comprised 65 (54.6\%) males and 54 (45.4\%) females. Milk feeding patterns differed from one infant to another and only 11 infants were exclusively breast fed prior to solid food introduction. Solid foods were introduced from four weeks to 17 weeks post-term. ${ }^{15}$

The prevalence of eczema (with or without other symptoms) at 12 months post-term was $35.8 \%$ in the entire cohort (table 3 ). Eczema alone occurred in $21.4 \%$ of the infants, while a further $14.4 \%$ had eczema with other symptoms; another $15.2 \%$ of infants had no eczema but other symptoms.

Of the 131 infants who either had eczema (with or without other symptoms) or who had no eczema but other symptoms at 12 months post-term, 75 (57.3\%) had an atopic background and $56(42.7 \%)$ had a non-atopic background. Of those infants who had no eczema and no other symptoms at 12 months post-term, $63(50 \%)$ had an atopic background and $63(50 \%)$ had a non-atopic background ( $\chi^{2}$ test, $p=0.224)$. Male infants had a statistically significant higher prevalence of allergic disease compared with female infants (83 (59.3\%) and $48(41.0 \%)$ respectively; $\chi^{2}$ test, $\left.p<0.004\right)$.

Investigation of the 34 sets of twins revealed that eight pairs $(23.5 \%)$ had eczema at 12 months post-term, 13 pairs $(38.2 \%)$ had no eczema, and 13 pairs $(38.2 \%)$ had one twin with eczema and one twin with no eczema. Of the two sets of triplets, one set had an infant with eczema and two infants with no eczema while the other set comprised three infants with no eczema.

\section{Statistical modelling of eczema (with or without other symptoms)}

Table 4 shows the outcome of the stepwise logistic regression analysis in the form of adjusted odds ratios with 95\% confidence intervals. No relation was found between any of the milk feeding groups and the development of eczema (with or without other symptoms) by 12 months post-term. However, the introduction of four or more solid foods at or before 17 weeks post-term was a significant risk factor. In addition, having non-atopic parents who introduced solid foods before 10 weeks post-term or having at least one atopic parent represented a significant risk for developing eczema (with or without other symptoms) by 12 months post-term. Early introduction of infant cereal was also a risk factor. Within the observed experience range of 5-50 weeks, an adjusted odds ratio of 1.07 was found for a delay of one week in introducing cereals. Hence, a two week delay would increase the odds ratio by a factor $1.07^{2}$ (i.e. 1.145), and a 12 week delay would increase the odds ratio by a factor of $1.07^{12}$ (i.e. 2.252).

When considering non-modifiable factors, being a male infant was a significant risk factor for the development of eczema (with or without other symptoms) by 12 months post-term. Table 5 shows variables found not to be statistically significantly related to the development of eczema (with or without other symptoms).

Table 2 Summary statistics for gestational age and birth weight of the 257 preterm infants according to their atopic background

\begin{tabular}{llllll}
\hline & Parents' atopic background & $\mathbf{n}$ & Mean & SD & Range \\
\hline Gestational age (weeks) & Atopic & 138 & 34.4 & 1.9 & $28-36$ \\
& Non-atopic & 119 & 34.4 & 1.7 & $28-36$ \\
Birth weight $(\mathrm{kg})$ & Atopic & 138 & 2.4 & 0.5 & $1.2-3.6$ \\
& Non-atopic & 119 & 2.3 & 0.5 & $1.0-3.4$ \\
\hline
\end{tabular}


Table 3 Prevalence of eczema (with or without other symptoms), no symptoms, and other symptoms diagnosed by 12 months post-term for 257 preterm infants

\begin{tabular}{|c|c|c|c|c|c|}
\hline \multicolumn{4}{|c|}{ Symptom group } & \multicolumn{2}{|c|}{ Prevalence } \\
\hline Eczema & $\begin{array}{l}\text { Lower respiratory } \\
\text { symptoms }\end{array}$ & $\begin{array}{l}\text { Upper respiratory } \\
\text { symptoms }\end{array}$ & $\begin{array}{l}\text { Gastrointestinal } \\
\text { symptoms }\end{array}$ & Number & Percentage \\
\hline Yes & No & No & No & 55 & 21.4 \\
\hline Yes & Yes & No & No & 17 & 6.6 \\
\hline Yes & No & Yes & No & 12 & 4.8 \\
\hline Yes & Yes & Yes & No & 5 & 1.9 \\
\hline Yes & No & No & Yes & 2 & 0.8 \\
\hline Yes & Yes & No & Yes & 1 & 0.4 \\
\hline No & No & No & No & 126 & 49.0 \\
\hline No & Yes & No & No & 22 & 8.6 \\
\hline No & Yes & Yes & No & 6 & 2.3 \\
\hline No & No & No & Yes & 6 & 2.3 \\
\hline No & No & Yes & No & 5 & 1.9 \\
\hline
\end{tabular}

\section{DISCUSSION}

\section{Study population}

Recruitment of the study population was undertaken in an affluent part of England with a strong commitment from the parents to the study, as measured by the low withdrawal rate (under $22 \%$ ) over the study period, which involved about 14 months for each family. The fact that the majority of parents were classified into socioeconomic groups I and II reflects the geographical area of recruitment; allergic disease is reported to be more frequent in affluent urban than in affluent rural groups. ${ }^{17}$ Although the parents were aware of the study objectives, they were not provided with specific advice on infant feeding guidelines; if this was requested, the study midwives provided general advice only.

Estimated prevalence of eczema in the UK's infant and child population is high. In a group of children aged 6-7 years in the north of England the prevalence of "atopic eczema" was $28 \% .{ }^{18}$ In the UK ISAAC study, $22.5 \%$ of the children aged $12-14$ years had eczema. ${ }^{19}$ In this cohort, $35.8 \%$ had eczema at 12 months post-term. Alhough this may be considered to be higher than expected, the prematurity of the cohort must be taken into consideration, together with factors such as early solid food introduction and the early introduction of a diversity of foods which also had important effects.

The local advisory committee did not permit SPT to be undertaken on the infants. This assessment is viewed by some as the "gold standard" for the identification of atopic disease, but we are unable to report here the numbers of positive or negative reactions. Because SPT were not performed, it was not possible to establish the numbers of infants where their eczema was associated with a non-IgE mediated food allergy. Delayed hypersensitivity (T lymphocyte mediated) to cows' milk typically manifests as skin reactions. ${ }^{20}$
A high prevalence of atopic disease was also apparent in the parents, with the atopic classification of over $30 \%$ of mothers and fathers leading to the presence of at least one atopic parent in over $50 \%$ of the families involved. This could partly be explained by the self-selecting nature of the study, where parents with an atopic nature might have been more likely to volunteer. However, the categorisation of atopic status was based on SPT, as well as an arbitrarily high serum IgE level and the results of a self administered questionnaire, thus resulting in a robust assessment of atopic status.

The practice of in vitro fertilisation and the fact that relatively more twins and triplets are born prematurely than singletons may explain the numbers of twins and triplets in the cohort. As the concordance of the twins was not established and the number of twins and triplets was relatively small, no meaningful conclusion could be drawn regarding their feeding habits and subsequent allergy development.

\section{Milk feeding practices}

Of the 11 infants who were exclusively breast fed until solid food introduction, five $(45.5 \%)$ had eczema at some point during the study. The complexity of milk feeding regimens was apparent from the groupings described in the Subjects and Methods section, and reflects the variety of ways in which infants are fed initially in neonatal care units in the UK. Others who have studied full term infants have reported a protective role for exclusive breast feeding in the development of atopic disease. ${ }^{41121}$ However it was not possible here to investigate the role of exclusive breast feeding in the prevention of allergic disorders for preterm infants, owing to the small number of infants experiencing this regimen.

Protective effect of delayed introduction of solid foods The third adjusted odds ratio category in table 4 highlights the opportunity for non-atopic parents to reduce the risk of

Table 4 Adjusted odds ratios with $95 \%$ confidence intervals for the development of eczema (with or without other symptoms) by 12 months post-term

\begin{tabular}{|c|c|c|c|c|}
\hline \multirow[b]{2}{*}{ Groups contrasted in adjusted odds ratio } & & \multirow{2}{*}{$\begin{array}{l}\text { Adjusted odds } \\
\text { ratio }\end{array}$} & \multicolumn{2}{|l|}{$95 \% \mathrm{Cl}$} \\
\hline & & & Lower limit & Upper limit \\
\hline Male, $n=140$ & Female, $\mathrm{n}=117$ & 1.84 & 1.04 & 3.27 \\
\hline Four or more foods by 17 weeks post-term, $n=203$ & Less than four foods by 17 weeks post-term, $n=54$ & 3.49 & 1.51 & 8.05 \\
\hline $\begin{array}{l}\text { Non-atopic parents with solid food introduction } \\
<10 \text { weeks post-term or any atopic parents, } n=162\end{array}$ & $\begin{array}{l}\text { Non-atopic parents with solid food introduction at } \\
\geqslant 10 \text { weeks post-term, } n=95\end{array}$ & 2.94 & 1.57 & 5.52 \\
\hline No central heating, $n=9$ & Central heating, $n=248$ & 6.37 & 1.31 & 30.90 \\
\hline Cereal introduced at $\mathrm{N}+1$ weeks from term & Cereal introduced at $\mathrm{N}$ weeks from term & 1.07 & 1.03 & 1.11 \\
\hline
\end{tabular}


Table 5 Variables found not to be statistically significant for the development of eczema (with or without other symptoms) by 12 months post-term

\section{Maternal age}

Single infant verses twin/triplet

Number of children less than 18 years old in the family

Maternal/paternal smoking at the time of recruitment

Presence of pets in home

Whether or not child had his/her own bedroom

Gestational age at birth

Birth weight

Total weeks of breast feeding

Number of weeks of breast feeding prior to solid food introduction

Whether or not ever breast fed

Whether or not exclusively breast fed before solid food introduction

Whether or not infant cows' milk formula used before solid food

introduction

Whether or not hypoallergenic formula used before solid food

introduction

Week at which cows' milk was introduced

Number of weeks from term cheese introduced

Number of weeks from term eggs introduced

Number of weeks from term fish introduced

Number of weeks from term fruit introduced

Number of weeks from term meat introduced

Number of weeks from term meat with vegetables introduced

Number of weeks from term baby rice introduced

Number of weeks from term baby rusks introduced

Number of weeks from term vegetables introduced

Number of weeks from term yoghurt introduced

Number of weeks from term other desserts introduced

the development of eczema (with or without other symptoms) during the first year by delaying the introduction of solid foods until at least 10 weeks post-term. However, this regimen has no corresponding benefit for families where one or both parents are atopic. The identification of this selective "risk reducing regimen" can be set in the context of evidence for the full term infant, where reducing the risk of the development of respiratory disease during the first seven years was associated with solid food introduction after 15 weeks from birth. ${ }^{11}$ As Zeiger and Heller showed, ${ }^{9}$ it is very difficult to moderate the later development of atopic disorders by early dietary manipulation. In that study, mothers in the intervention group avoided cows' milk, egg, and peanut in the last trimester and lactation. In addition, the diet of the full term infants was manipulated so that they avoided cows' milk to 12 months, egg to 2 years, and peanuts to 3 years. However, the results were disappointing when the cohort was followed to 7 years, as allergy associated with foods, atopic dermatitis, allergic rhinitis, and asthma did not differ between the standard and intervention feeding groups, although significant differences were reported at 2 years. The authors suggested that the diet intervention was probably too stringent and impractical for many mothers.

\section{Age of introduction of solid foods to the preterm infant}

High dietary needs for energy and iron are not met in the preterm infant from breast milk alone after a certain point in time, although a suitably fortified post-discharge cows' milk infant formula may overcome the nutritional deficiencies. A study of weaning practices in the UK in the $1990 \mathrm{~s}^{22}$ has shown that $67 \%$ and $82 \%$ of breast fed and formula fed preterm infants respectively were receiving solid foods by 12 weeks from term. In contrast, Marriott and colleagues ${ }^{23}$ have reported in a small randomised controlled study of 68 preterm infants that early ( 12 weeks from birth) nutrient enriched solid feeding was associated with enhanced length growth and iron status at 12 months post-term. In that study no information has been published regarding subsequent development of eczema. Here we have shown a benefit in eczema outcome to the high risk preterm infant of waiting to introduce solids to 10 weeks from term, though any detrimental effects on growth or micronutrient status have not yet been established.

The practical implementation of our findings should be viewed with caution, especially for those born at 34 weeks gestational age and earlier because of their high energy and nutrient needs. ${ }^{24}$ Currently, official recommendations on the introduction of solid foods to the preterm infant (based on weight attainment of $5 \mathrm{~kg}^{25}$ ) are not universally followed..$^{15}$ There is no consensus for such recommendations and they are not evidence based. In the development of feeding guidelines for the preterm infant, there will inevitably be tensions between the early introduction of solid foods, which will benefit rapid (catch up) growth, and the late introduction of certain solid foods, which will protect against eczema.

\section{Type and frequency of solid food introduction}

The early introduction of a diversified range of four or more solid foods before 17 weeks post-term was shown to be associated with a higher risk of eczema (with or without other symptoms) at 12 months post-term in our cohort. This phenomenon has been observed in the full term infant ${ }^{12}$ where the provision of four foods or more from 17 weeks of age was associated with an increased risk of the development of recurrent or chronic eczema at the age of 10 years. There is little consensus on the first type of solid food to introduce, but low allergen foods such as potatoes, baby rice, fruit, and vegetables have been advocated. ${ }^{26}$ Here the first food was predominantly a low allergenic food: baby rice. ${ }^{15}$ However, for those infants where early introduction of multiple foods was practised, a significantly higher predisposition to eczema development was observed.

Since the gluten content in cereal was not specified, the finding that the "late" introduction of cereal was a risk factor in the development of eczema (with or without other symptoms) should be interpreted with caution. The relation was weaker than for the other variables in the model as shown by the lower $95 \%$ confidence interval being close to 1.00. That the introduction of baby rice was not found to be beneficial in protecting against eczema development may again be explained by the lack of a clear definition of cereals containing gluten.

\section{Gender and central heating effects}

Male infants were at greater risk than female infants of being diagnosed with eczema (with or without other symptoms) at 12 months post-term. The gender effect is an unmodifiable but well recognised factor associated with risk of atopy. ${ }^{9}$ As central heating was not specifically defined (no distinction was made between gas, oil, and electricity) and most households had central heating of some sort, the finding that its absence was a risk factor in the development of eczema may be of little practical importance when set in the context of the other findings. An examination of the descriptors for nine families without central heating revealed no specific common factor to explain the finding (for example, if all families were atopic or all parents smoked). This finding deserves further investigation.

\section{Conclusions}

We have established that the development of eczema in the preterm infant is influenced by certain solid feeding practices, and these practices are generally in concert with those for the full term infant - that is, to delay solid foods and to introduce single food components initially. Male infants and those with an atopic background were at greater risk of the development of eczema than the rest. 
Future recommendations need to be evidence based. They should identify optimal solid food diets, for example, specific foods with appropriate macro- and micronutrient status. Any recommendation should be based on appropriate growth as well as positive health outcomes.

\section{ACKNOWLEDGEMENTS}

The authors wish to thank the Food Intolerance Programme, Foods Standards Agency, for the generous support of this study, and Cow \& Gate Nutricia, Trowbridge, Wilts, for the supply of Pepti Junior hypoallergenic formula. We also thank Dr M Fewtrell, Professor JWT Dickerson, Dr AC Kimber, and Professor S Strobel for their invaluable comments on the draft manuscript.

\section{Authors' affiliations}

J Morgan, F Norris, C M Williams, M Larkin, S Hampton, School of Biomedical and Life Sciences, University of Surrey, Guildford, Surrey, GU2 7XH, UK

P Williams, Department of Mathematics and Statistics, University of Surrey, Guildford, Surrey, GU2 7XH, UK

\section{REFERENCES}

1 Host A, Koletzko B, Dreborg S, et al. Dietary product used in infants for treatment and prevention of food allergy. Arch Dis Child 1999:81:80-4.

2 Warner JO, Jones CA, Kilburn SA, et al. Pre-natal sensitisation in humans. Pediatr Allergy Immunol 2000;13(suppl):6-8.

3 Cant AJ, Bailes JA, Marsden RA, et al. Effect of maternal dietary exclusion on breast fed infants with eczema: two controlled studies. BMJ 1986;293:231-3.

4 Arshad SH, Matthews S, Gant C, et al. Effect of allergy avoidance on development of allergic disorders in infancy. Lancet 1992;339:1494-7.

5 Sigurs N, Hattevig G, Kjellman B. Maternal avoidance, cow's milk and fish during lactation: effect on allergic manifestations, skin prick tests and specific lgE antibodies in children at age 4 years. Pediatrics 1992;89:735-9.

6 Lilija G, Dannaeus A, Foucard T, et al. Effects of maternal diet during late pregnancy and lactation on the development of atopic disease in infants up to 18 months of age - in vivo results. Clin Exp Allergy 1989;19:473-9.

7 Lovegrove JA, Hampton SM, Morgan JB. The immunological and long term atopic outcome in infants born to women following a milk-free diet during late pregnancy and lactation: a pilot study. Br J Nutr 1994;71:223-38.

8 Marini A, Agosti M, Motta G, et al. Effects of a dietary and environmental prevention programme on the incidence of allergy symptoms in high atopic risk infants: three year follow-up. Acta Paediatr 1996;414(suppl): 1-22.
9 Zeiger RS, Heller S. The development and prediction of atopy in high-risk children: Follow-up at age seven years in a prospective randomised study of combined maternal and infant food allergen avoidance. J Allergy Clin Immunol 1995;95:1179-90.

10 Kajaosaari M, Saarien UM. Prophylaxis of atopic disease by six months' total solid food elimination. Evaluation of 135 exclusively breast-fed infants of atopic families. Acta Paediatr Scand 1983;72:411-14.

11 Wilson AC, Stewart Forsyth J, Greene SA, et al. Relation of infant diet to childhood health: seven year follow up of cohort of children in Dundee infant feeding study. BMJ 1998;316:21-5.

12 Fergusson DM, Horwood $\amalg$, Sannon FT. Early solid feeding and recurrent childhood eczema: a 10 year longitudinal study. Pediatrics 1990;86:541-6.

13 Chandra RK. Nutrition and the immune system from birth to old age. Eur J Clin Nutr 2002;56(suppl 3):S73-6.

14 Norris FJ, Williams CM, Larkin $M$, et al. Total lgE and specific lgG levels to $\beta$ lactoglobulin and ovalbumin and allergy outcome in small for gestational age infants - a pilot study. Pediatr Asthma Allergy Immunol 1999;13:171-7.

15 Norris FJ, Williams CM, Larkin $M$, et al. Factors affecting the introduction of complementary feeding in the preterm infant. Eur J Clin Nutr 2002; 56:448-54

16 ETAC Study Group. Allergic factors associated with the development of asthma and the influence of cetirizine in a double blind, randomised controlled trial: first results of ETAC. Pediatr Allergy Immunol 1998;9:1 16-24.

17 Derereux G. Epidemiology of food intolerance and food allergy. In: Buttriss J, ed. Adverse reactions to foods. Oxford: Blackwell Science, 2002.

18 Shamssain MH, Shamsian N. Prevalence and severity of asthma, rhinitis, and atopic eczema: the north east study. Arch Dis Child 1999;81:313-17.

19 Austin JB, Kaur B, Anderson HR, et al. Hay fever, eczema and wheeze: a nationwide UK study (ISAAC-International Study of Asthma and Allergies in Childhood). Arch Dis Child 1999;81:225-30.

20 Lessof M. Common food allergies. In: Buttriss J, ed. Adverse reactions to foods. Oxford: Blackwell Science, 2002.

21 Oddy WH, Holt PG, Sly PD, et al. Association between breast feeding and asthma in 6 year old children: findings of a prospective birth cohort study. BMJ 1999;319:815-19.

22 Fewtrell M, Lucas A, Morgan JB. Is the age of weaning infant lead or mother lead? Factors associated with the introduction of solid foods in the full term and preterm infant. Arch Dis Child Fetal Neonatal Ed 2003;88:F296-301.

23 Marriott LM, Foote K, Bishop JA, et al. Weaning preterm infants: a randomised controlled trial. Arch Dis Child Fetal Neonatal Ed 2003;88:F302-7

24 Tsang RC, Lucas A, Uauy R, et al. Consensus recommendations. In: Tsang RC Lucas A, Uauy R, Zlotkin S, eds. Nutritional needs of the preterm infant. Scientific basis and practical guidelines. Baltimore: Williams and Wilkins, 1993.

25 Department of Health. Weaning and the weaning diet. Report on Health and Social Subjects no. 45. London: HMSO, 1994.

26 Kemeny M, MacDonald A, Kimber I. Pre- and post sensitisation to foods. In: Buttriss J, ed. Adverse reactions to foods. Oxford: Blackwell Science, 2002.

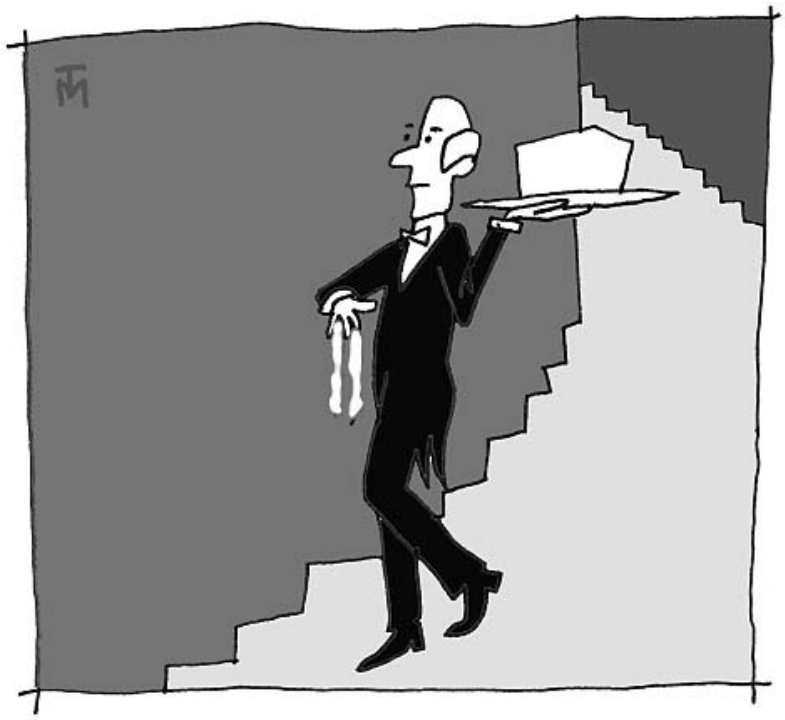

